Vietnam Journal of Mechanics, VAST, Vol.39, No. 4 (2017), pp. 365-374

DOI:10.15625/0866-7136/10033

\title{
APPROXIMATE FORMULA FOR THE H/V RATIO OF RAYLEIGH WAVES IN INCOMPRESSIBLE ORTHOTROPIC HALF-SPACES COATED BY A THIN ELASTIC LAYER
}

\author{
Pham Chi Vinh ${ }^{1, *}$, Tran Thanh Tuan ${ }^{1}$, Le Thi Hue ${ }^{2}$ \\ ${ }^{1}$ VNU University of Science, Hanoi, Vietnam \\ ${ }^{2}$ Vietnam National University of Forestry, Hanoi, Vietnam \\ *E-mail: pcvinh@vnu.edu.vn \\ Received June 10, 2017
}

\begin{abstract}
This paper is concerned with the propagation of Rayleigh waves in an incompressible orthotropic elastic half-space coated with a thin incompressible orthotropic elastic layer. The main purpose of the paper is to establish an approximate formula for the Rayleigh wave H/V ratio (the ratio between the amplitudes of the horizontal and vertical displacements of Rayleigh waves at the traction-free surface of the layer). First, the relations between the traction amplitude vector and the displacement amplitude vector of Rayleigh waves at two sides of the interface between the layer and the half-space are created using the Stroh formalism and the effective boundary condition method. Then, an approximate formula for the Rayleigh wave H/V ratio of third-order in terms of dimensionless thickness of the layer has been derived by using these relations along with the Taylor expansion of the displacement amplitude vector of the thin layer at its traction-free surface. It is shown numerically that the obtained formula is a good approximate one. It can be used for extracting mechanical properties of thin films from measured values of the Rayleigh wave $\mathrm{H} / \mathrm{V}$ ratio.

Keywords: Rayleigh waves, the Rayleigh wave H/V ratio, incompressible orthotropic elastic half-space, thin incompressible orthotropic elastic layer, approximate formula for the Rayleigh wave $\mathrm{H} / \mathrm{V}$ ratio.
\end{abstract}

\section{INTRODUCTION}

As addressed by Junge et al. [1], the H/V ratio (the ratio between the amplitudes of the horizontal and vertical displacements of Rayleigh waves at the traction-free surface of half-spaces) is more sensitive than the Rayleigh wave velocity as an indicator of the state of stress, and, in contrast to the Rayleigh wave velocity, it is reference free. Therefore, formulas for the Rayleigh wave $\mathrm{H} / \mathrm{V}$ ratio are a powerful tool for nondestructively evaluating prestress of structures before and during loading. The Rayleigh wave H/V ratio is also an important parameter which reflects fundamental properties of the elastic

(C) 2017 Vietnam Academy of Science and Technology 
material [2]. It can be thus used for the nondestructive evaluation of the elastic constants of material [3], besides its well-known applications in seismology [4,5].

While a large number of formulas for the Rayleigh wave velocity have been derived, see for examples, [6-16], only few formulas for the Rayleigh wave H/V ratio have been obtained. They are, for example, the exact Rayleigh wave $\mathrm{H} / \mathrm{V}$ ratio formula for a compressible layered half-space with traction-free surface [2], the exact and an approximate formula for that model of incompressible media [17]. However, these formulas are only for the isotropic media.

In this paper, an approximate formula of third order for the Rayleigh wave H/V ratio has been established for an incompressible orthotropic elastic half-space coated by a thin incompressible orthotropic elastic layer. This formula is derived by using the relations between the traction and displacement amplitude vectors of Rayleigh waves at two sides of the welded interface between the layer and the half-space, along with the Taylor expansion of the displacement amplitude vector of the thin layer at its traction-

free surface. It is shown numerically that the obtained approximate formula is a good approximation.

\section{RELATIONS BETWEEN THE TRACTION AND DISPLACEMENT AMPLITUDE VECTORS AT TWO SIDES OF THE INTERFACE}

\subsection{Basic equations for an incompressible orthotropic elastic layer in matrix form}

Consider an elastic half-space $x_{2} \geq 0$ coated by a thin elastic layer $-h \leq x_{2} \leq 0$. Both the layer and half-space are assumed to be incompressible, orthotropic and they are in welded contact with each other. Note that same quantities related to the half-space and the layer have the same symbol but are systematically distinguished by a bar if pertaining to the layer. We are interested in the plain strain so that

$$
u_{i}=u_{i}\left(x_{1}, x_{2}, t\right), \quad \bar{u}_{i}=\bar{u}_{i}\left(x_{1}, x_{2}, t\right), \quad i=1,2, \quad u_{3}=\bar{u}_{3} \equiv 0,
$$

where $t$ is the time. Since the material of the layer is incompressible and orthotropic, the strain-stress relations are [8]

$$
\bar{\sigma}_{11}=-\bar{p}+\bar{c}_{11} \bar{u}_{1,1}+\bar{c}_{12} \bar{u}_{2,2}, \quad \bar{\sigma}_{22}=-\bar{p}+\bar{c}_{12} \bar{u}_{1,1}+\bar{c}_{22} \bar{u}_{2,2}, \quad \bar{\sigma}_{12}=\bar{c}_{66}\left(\bar{u}_{1,2}+\bar{u}_{2,1}\right),
$$

where $\bar{\sigma}_{i j}, \bar{p}$ and $\bar{c}_{i j}$ are respectively the stress, the hydrostatic pressure associated with the incompressibility constraint and the material constants, commas indicate differentiation with respect to the spatial variables $x_{k}$. In the absence of body forces, the equations of motion are

$$
\bar{\sigma}_{11,1}+\bar{\sigma}_{12,2}=\bar{\rho} \ddot{\bar{u}}_{1}, \quad \bar{\sigma}_{12,1}+\bar{\sigma}_{22,2}=\bar{\rho} \ddot{\bar{u}}_{2},
$$

where $\bar{\rho}$ is the mass density, a dot signifies differentiation with respect to the time $t$. The incompressibility gives

$$
\bar{u}_{1,1}+\bar{u}_{2,2}=0 \text {. }
$$

From (1)-(4) we have

$$
\left[\begin{array}{c}
\overline{\mathbf{u}}^{\prime} \\
\overline{\mathbf{t}}^{\prime}
\end{array}\right]=\left[\begin{array}{ll}
M_{1} & M_{2} \\
M_{3} & M_{1}
\end{array}\right]\left[\begin{array}{c}
\overline{\mathbf{u}} \\
\overline{\mathbf{t}}
\end{array}\right],
$$


where $\overline{\mathbf{u}}=\left[\begin{array}{ll}\bar{u}_{1} & \bar{u}_{2}\end{array}\right]^{T}, \overline{\mathbf{t}}=\left[\begin{array}{ll}\bar{\sigma}_{12} & \bar{\sigma}_{22}\end{array}\right]^{T}$, the symbol “T" indicates the transpose of a matrix, the prime signifies the derivative with respect to $x_{2}$ and

$$
M_{1}=\left[\begin{array}{cc}
0 & -\partial_{1} \\
-\partial_{1} & 0
\end{array}\right], M_{2}=\left[\begin{array}{cc}
\frac{1}{\bar{c}_{66}} & 0 \\
0 & 0
\end{array}\right], M_{3}=\left[\begin{array}{cc}
-\bar{\delta} \partial_{1}^{2}+\bar{\rho} \partial_{t}^{2} & 0 \\
0 & \bar{\rho} \partial_{t}^{2}
\end{array}\right],
$$

here we use the notations $\partial_{1}=\partial / \partial x_{1}, \partial_{1}^{2}=\partial^{2} / \partial x_{1}^{2}, \partial_{t}^{2}=\partial^{2} / \partial x_{t}^{2}$. Eq. (5) is called the matrix equation for an incompressible orthotropic elastic layer in plane strain.

\subsection{Stroh formalism for an incompressible orthotropic elastic layer}

Now we consider the propagation of a plane wave traveling in the $x_{1}$-direction with velocity $c(>0)$ and wave number $k(>0)$. Then, displacement components of the wave are sought in the form

$$
\bar{u}_{n}=\bar{U}_{n}(y) e^{i k\left(x_{1}-c t\right)}, \quad \bar{\sigma}_{n 2}=i k \bar{\Sigma}_{n}(y) e^{i k\left(x_{1}-c t\right)}, n=1,2, y=k x_{2} .
$$

Substituting (7) into (5) yields

$$
\xi^{\prime}=i N \xi, y \in[-\varepsilon, 0], \varepsilon=k h,
$$

where the prime signifies differentiation with respect to $y$ and

$$
\xi=\left[\begin{array}{l}
\bar{U} \\
\bar{\Sigma}
\end{array}\right], \quad \bar{U}=\left[\begin{array}{l}
\bar{U}_{1} \\
\bar{U}_{2}
\end{array}\right], \quad \bar{\Sigma}=\left[\begin{array}{l}
\bar{\Sigma}_{1} \\
\bar{\Sigma}_{2}
\end{array}\right], \quad N=\left[\begin{array}{ll}
N_{1} & N_{2} \\
N_{3} & N_{4}
\end{array}\right]
$$

in which the matrices $N_{k}$ are given by

$$
N_{1}=\left[\begin{array}{cc}
0 & -1 \\
-1 & 0
\end{array}\right], N_{2}=\left[\begin{array}{cc}
\frac{1}{\bar{c}_{66}} & 0 \\
0 & 0
\end{array}\right], N_{3}=\left[\begin{array}{cc}
-\bar{\delta}+\bar{\rho} c^{2} & 0 \\
0 & \bar{\rho} c^{2}
\end{array}\right], N_{4}=N_{1}
$$

where $\bar{\delta}=\bar{c}_{11}+\bar{c}_{22}-2 \bar{c}_{12}$. Eq. (8) is called the Stroh formalism [18] for an incompressible orthotropic elastic layer. From (8) it follows

$$
\xi^{(n)}=i^{n} N^{n} \xi, N^{n}:=\left[\begin{array}{ll}
N_{1}^{(n)} & N_{2}^{(n)} \\
N_{3}^{(n)} & N_{4}^{(n)}
\end{array}\right], y \in\left[\begin{array}{ll}
-\varepsilon, 0
\end{array}\right] .
$$

\subsection{Relations between the traction and displacement amplitude vectors at two sides of the interface}

Let $\varepsilon:=k h$ be small (i.e., the layer is thin) and the surface $x_{2}=-h$ of the layer is free of traction: $\bar{\Sigma}(-\varepsilon)=0$. By expanding into Taylor series $\bar{\Sigma}(-\varepsilon)$ at $y=0$ up to the third-order and using (11) along with $\bar{\Sigma}(-\varepsilon)=0$ we arrive at the relation between the traction and displacement amplitude vectors at the layer-side of the interface $y=0$, namely

$$
A \bar{U}(0)+B \bar{\Sigma}(0)=0
$$


where matrices $A$ and $B$ are given by

$$
\begin{aligned}
& A=\left[\begin{array}{cc}
-i\left[\varepsilon\left(\bar{\rho} c^{2}-\bar{\delta}\right)-\frac{\varepsilon^{3}}{6}\left(r_{2}+r_{3} \bar{\rho} c^{2}+\frac{\bar{\rho}^{2} c^{4}}{\bar{c}_{66}}\right)\right] & \frac{\varepsilon^{2}}{2}\left[2 \bar{\rho} c^{2}-\bar{\delta}\right] \\
\frac{\varepsilon^{2}}{2}\left[2 \bar{\rho} c^{2}-\bar{\delta}\right] & -i\left[\varepsilon \bar{\rho} c^{2}-\frac{\varepsilon^{3}}{6}\left\{3 \bar{\rho} c^{2}-\bar{\delta}\right\}\right]
\end{array}\right], \\
& B=\left[\begin{array}{cc}
1-\frac{\varepsilon^{2}}{2}\left(r_{1}+\frac{\bar{\rho} c^{2}}{\bar{c}_{66}}\right) & i \varepsilon \\
i \varepsilon & 1-\frac{\varepsilon^{2}}{2}
\end{array}\right],
\end{aligned}
$$

where

$$
r_{1}=1-\frac{\bar{\delta}}{\bar{c}_{66}}, \quad r_{2}=\bar{\delta}\left(\frac{\bar{\delta}}{\bar{c}_{66}}-2\right), \quad r_{3}=2 r_{1}+1 .
$$

According to Ogden and Vinh [8], the displacement components of Rayleigh waves in the half-space are given by

$$
u_{n}=U_{n}(y) e^{i k\left(x_{1}-c t\right)}, \quad \sigma_{n 2}=i k \Sigma_{n}(y) e^{i k\left(x_{1}-c t\right)}, n=1,2
$$

where

$$
\begin{gathered}
U_{1}(y)=\alpha_{1} B_{1} e^{-b_{1} y}+\alpha_{2} B_{2} e^{-b_{2} y}, \quad U_{2}(y)=i\left(B_{1} e^{-b_{1} y}+B_{2} e^{-b_{2} y}\right), \\
\Sigma_{1}(y)=i\left(\beta_{1} B_{1} e^{-b_{1} y}+\beta_{2} B_{2} e^{-b_{2} y}\right), \quad \Sigma_{2}(y)=\gamma_{1} B_{1} e^{-b_{1} y}+\gamma_{2} B_{2} e^{-b_{2} y},
\end{gathered}
$$

here $\alpha_{k}, \beta_{k}, \gamma_{k}$ are defined by

$$
\begin{aligned}
& \alpha_{k}=b_{k}, \beta_{k}=c_{66}\left(1+\alpha_{k}^{2}\right), \gamma_{k}=\left(X-\delta+\beta_{k}\right) \alpha_{k}, k=1,2 \\
& X=\rho c^{2}, \quad \delta=c_{11}+c_{22}-2 c_{12},
\end{aligned}
$$

and $b_{1}, b_{2}$ are to with positive real parts roots of the equation

$$
c_{66} b^{4}-\left(\delta-2 c_{66}-X\right) b^{2}+\left(c_{66}-X\right)=0 .
$$

From (19) we have

$$
\begin{aligned}
& b_{1}^{2}+b_{2}^{2}=\frac{\delta-2 c_{66}-X}{c_{66}}:=S, \\
& b_{1}^{2} \cdot b_{2}^{2}=\frac{c_{66}-X}{c_{66}}:=P .
\end{aligned}
$$

It is not difficult to verify that if a Rayleigh wave exists $\left(\rightarrow b_{1}, b_{2}\right.$ having positive real parts), then

$$
0<X<c_{66}, P>0, S+2 \sqrt{P}>0, b_{1} \cdot b_{2}=\sqrt{P}, b_{1}+b_{2}=\sqrt{S+2 \sqrt{P}} .
$$

Taking $x_{2}=0$ in (16) and (17) gives

$$
\begin{aligned}
& U_{1}(0)=\alpha_{1} B_{1}+\alpha_{2} B_{2}, U_{2}(0)=i\left(B_{1}+B_{2}\right), \\
& \Sigma_{1}(0)=i\left(\beta_{1} B_{1}+\beta_{2} B_{2}\right), \Sigma_{2}(0)=\gamma_{1} B_{1}+\gamma_{2} B_{2} .
\end{aligned}
$$


Eliminating $B_{1}, B_{2}$ from Eqs. (22) we have

$$
\Sigma(0)=H U(0), \quad H=\left[\begin{array}{cc}
i h_{11} & h_{12} \\
-h_{12} & -i h_{22}
\end{array}\right],
$$

where

$$
h_{11}=c_{66}\left(b_{1}+b_{2}\right), h_{12}=c_{66}\left(1-b_{1} b_{2}\right), h_{22}=-c_{66} b_{1} b_{2}\left(b_{1}+b_{2}\right),
$$

and $b_{1} b_{2}, b_{1}+b_{2}$ are defined by (21). Eq. (23) is the relation between the traction and displacement amplitude vectors at the half-space-side of the interface $y=0$.

\section{AN APPROXIMATE FORMULAS FOR THE RAYLEIGH WAVE H/V RATIO}

Suppose the layer and the half-space are perfectly bonded at the interface $y=0$, then we have

$$
\bar{U}(0)=U(0), \bar{\Sigma}(0)=\Sigma(0) .
$$

Expanding into Taylor series $\bar{U}(-\varepsilon)$ at $y=0$ up to the third order yields

$$
\bar{U}(-\varepsilon)=\bar{U}(0)-\varepsilon \bar{U}^{\prime}(0)+\frac{\varepsilon^{2}}{2} \bar{U}^{\prime \prime}(0)-\frac{\varepsilon^{3}}{6} \bar{U}^{\prime \prime \prime}(0) .
$$

From (11), (25) and the relation between the traction and displacement amplitude vectors at the half-space-side of the interface (23), it follows

$$
\begin{aligned}
& \bar{U}^{\prime}(0)=i\left(N_{1}+N_{2} H\right) U(0), \bar{U}^{\prime \prime}(0)=i^{2}\left(N_{1}^{(2)}+N_{2}^{(2)} H\right) U(0), \\
& \bar{U}^{\prime \prime \prime}(0)=i^{3}\left(N_{1}^{(3)}+N_{2}^{(3)} H\right) U(0) .
\end{aligned}
$$

Substituting (27) into (26) leads to

$$
\bar{U}(-\varepsilon)=Q U(0) .
$$

Elements of matrix $Q$ in (28) are defined by

$$
\begin{aligned}
Q_{11}= & +\varepsilon\left\{\frac{h_{11}}{\bar{c}_{66}}\right\}-\frac{\varepsilon^{2}}{2}\left\{r_{1}+\frac{\bar{\rho} c^{2}}{\bar{c}_{66}}+\frac{h_{12}}{\bar{c}_{66}}\right\} \\
& -\frac{\varepsilon^{3}}{6}\left\{\left(\frac{1+r_{1}}{\bar{c}_{66}}+\frac{\bar{\rho} c^{2}}{\bar{c}_{66}^{2}}\right) h_{11}\right\}, \\
Q_{12}= & i\left[\varepsilon\left\{1-\frac{h_{12}}{\bar{c}_{66}}\right\}-\frac{\varepsilon^{2}}{2}\left\{\frac{h_{22}}{\bar{c}_{66}}\right\}\right. \\
& \left.-\frac{\varepsilon^{3}}{6}\left\{r_{1}+\frac{2 \bar{\rho} c^{2}}{\bar{c}_{66}}-\left(\frac{1+r_{1}}{\bar{c}_{66}}+\frac{\bar{\rho} c^{2}}{\bar{c}_{66}^{2}}\right) h_{12}\right\}\right], \\
Q_{21}= & i\left[\varepsilon+\frac{\varepsilon^{2}}{2}\left\{\frac{h_{11}}{\bar{c}_{66}}\right\}-\frac{\varepsilon^{3}}{6}\left\{r_{1}+\frac{\bar{\rho}^{2}}{\bar{c}_{66}}+\frac{h_{12}}{\bar{c}_{66}}\right\}\right], \\
Q_{22}= & 1+\frac{\varepsilon^{2}}{2}\left\{-1+\frac{h_{12}}{\bar{c}_{66}}\right\}+\frac{\varepsilon^{3}}{6}\left\{\frac{h_{22}}{\bar{c}_{66}}\right\} .
\end{aligned}
$$

On the other hand, using (12), (23), and taking into account (25) yield

$$
Z U(0)=0, \quad Z=A+B H .
$$


Elements of matrix $Z$ in (30) are defined by

$$
\begin{aligned}
Z_{11}= & i\left[h_{11}+\varepsilon\left\{\bar{\delta}-\bar{\rho} c^{2}-h_{12}\right\}-\frac{\varepsilon^{2}}{2}\left\{\left(r_{1}+\frac{\bar{\rho} c^{2}}{\bar{c}_{66}}\right) h_{11}\right\}\right. \\
& +\frac{\varepsilon^{3}}{6}\left\{r_{2}+r_{3} \bar{\rho} c^{2}+\frac{\bar{\rho}^{2} c^{4}}{\bar{c}_{66}}\right\}, \\
Z_{12}= & h_{12}+\varepsilon h_{22}+\frac{\varepsilon^{2}}{2}\left\{2 \bar{\rho} c^{2}-\bar{\delta}-\left(r_{1}+\frac{\bar{\rho} c^{2}}{\bar{c}_{66}}\right) h_{12}\right\}, \\
Z_{21}= & -h_{12}-\varepsilon h_{11}+\frac{\varepsilon^{2}}{2}\left\{2 \bar{\rho} c^{2}-\bar{\delta}+h_{12}\right\}, \\
Z_{22}= & i\left[-h_{22}+\varepsilon\left\{-\bar{\rho} c^{2}+h_{12}\right\}+\frac{\varepsilon^{2}}{2} h_{22}+\frac{\varepsilon^{3}}{6}\left\{-\bar{\delta}+3 \bar{\rho} c^{2}\right\}\right] .
\end{aligned}
$$

From (28) and (30), it follows

$$
\chi:=\left|\frac{\bar{u}_{1}(-h)}{\bar{u}_{2}(-h)}\right|=\left|\frac{\bar{U}_{1}(-\varepsilon)}{\bar{U}_{2}(-\varepsilon)}\right|=\left|\frac{Q_{12} Z_{11}-Q_{11} Z_{12}}{Q_{22} Z_{11}-Q_{21} Z_{12}}\right|,
$$

where $Z_{11}, Z_{12}$ are defined by (31), elements of matrix $Q$ are defined by (29). Note that, due to $|Z|=0$, therefore $\chi$ can be given by an alternative formula

$$
\chi:=\left|\frac{Q_{12} Z_{21}-Q_{11} Z_{22}}{Q_{22} Z_{21}-Q_{21} Z_{22}}\right| .
$$

After some manipulations, we arrive at the desired approximate formula of third order for the $\mathrm{H} / \mathrm{V}$ ratio, namely

$$
\chi=\left|\frac{A_{0}+A_{1} \varepsilon+A_{2} \frac{\varepsilon^{2}}{2}+A_{3} \frac{\varepsilon^{3}}{6}+O\left(\varepsilon^{4}\right)}{B_{0}+B_{1} \varepsilon+B_{2} \frac{\varepsilon^{2}}{2}+B_{3} \frac{\varepsilon^{3}}{6}+O\left(\varepsilon^{4}\right)}\right|,
$$

where

$$
\begin{aligned}
& A_{0}=-h_{12}, A_{1}=-h_{11}-h_{22}, \\
& A_{2}=-\bar{\delta}+2\left(1+r_{1}+\frac{\bar{\delta}}{\bar{c}_{66}}\right) h_{12}-\frac{1}{\bar{c}_{66}}\left(h_{12}^{2}+h_{11} h_{22}\right), \\
& A_{3}=3\left(r_{1}+\frac{\bar{\delta}}{\bar{c}_{66}}\right) h_{22}+\left(4 r_{1}+\frac{3 \bar{\delta}}{\bar{c}_{66}}-\frac{\bar{\rho} c^{2}}{\bar{c}_{66}}\right) h_{11},
\end{aligned}
$$

and

$$
\begin{aligned}
B_{0}= & h_{11}, B_{1}=\bar{\delta}-\bar{\rho} c^{2}-2 h_{12}, \\
B_{2}= & -2 h_{22}-\left(1+r_{1}+\frac{\bar{\rho} c^{2}}{\bar{c}_{66}}\right) h_{11}, \\
B_{3}= & r_{2}+\left(r_{3}-3\right) \bar{\rho} c^{2}+\frac{\bar{\rho}^{2} c^{4}}{\bar{c}_{66}}+\left(3+4 r_{1}+\frac{3 \bar{\delta}}{\bar{c}_{66}}+\frac{\bar{\rho} c^{2}}{\bar{c}_{66}}\right) h_{12} \\
& -\frac{2}{\bar{c}_{66}}\left(h_{12}^{2}+h_{11} h_{22}\right) .
\end{aligned}
$$


In the dimensionless form Eq. (34) is of the form

$$
\chi=\left|\frac{\bar{A}_{0}+\bar{A}_{1} \varepsilon+\bar{A}_{2} \frac{\varepsilon^{2}}{2}+\bar{A}_{3} \frac{\varepsilon^{3}}{6}+O\left(\varepsilon^{4}\right)}{\bar{B}_{0}+\bar{B}_{1} \varepsilon+\bar{B}_{2} \frac{\varepsilon^{2}}{2}+\bar{B}_{3} \frac{\varepsilon^{3}}{6}+O\left(\varepsilon^{4}\right)}\right|,
$$

in which the coefficients $\bar{A}_{k}, \bar{B}_{k}(k=0,1,2,3)$ are given by (47) in Appendix A and they depend on the following dimensionless parameters

$$
e_{\delta}=\frac{\delta}{c_{66}}, \quad \bar{e}_{\delta}=\frac{\bar{\delta}}{\bar{c}_{66}}, \quad r_{\mu}=\frac{\bar{c}_{66}}{c_{66}}, \quad r_{v}=\frac{c_{2}}{\bar{c}_{2}}, \quad x=\frac{X}{c_{66}},
$$

where $c_{2}=\sqrt{c_{66} / \rho}, \bar{c}_{2}=\sqrt{\bar{c}_{66} / \bar{\rho}}$. It is clear that the H/V ratio $\chi$ depends on 5 dimensionless parameters: $e_{\delta}, \bar{e}_{\delta}, r_{\mu}, r_{v}$ and $\varepsilon$ which are subjected the inequalities [19]

$$
r_{\mu}>0, \quad r_{v}>0, \quad e_{\delta}>0, \quad \bar{e}_{\delta}>0, \quad \varepsilon>0 .
$$

Note that the Rayleigh wave H/V ratio $\chi$ depends on the dimensionless Rayleigh wave velocity $x$ that is a solution of the secular equation (3.14) in [19] and it depends also on 5 dimensionless parameters mentioned above.

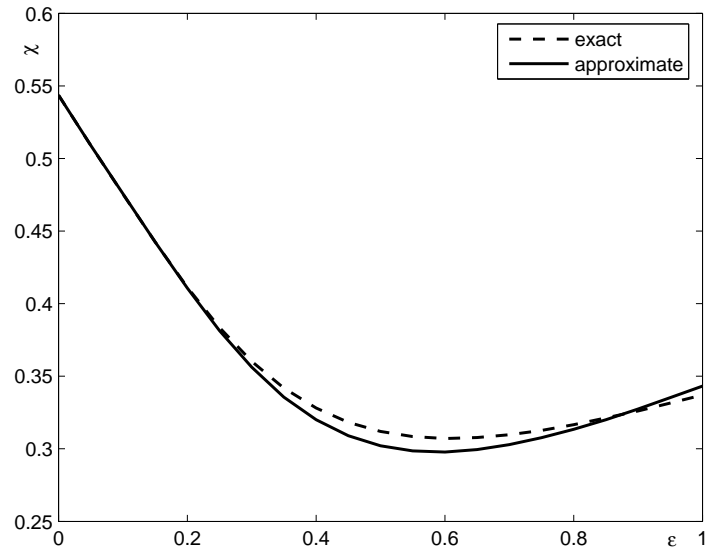

Fig. 1. Dependence of the $\mathrm{H} / \mathrm{V}$ ratio on $\varepsilon \in$ $[0,1]$ that is calculated by the exact formula (dashed line), by the third-order approximate formula (37) (solid line). Here we take:

$$
e_{\delta}=\bar{e}_{\delta}=4, r_{\mu}=1, r_{v}=3
$$

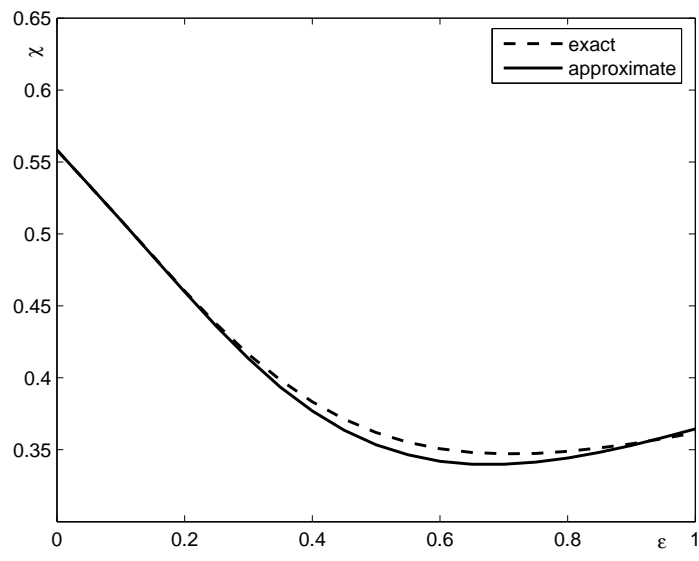

Fig. 2. Dependence of the $\mathrm{H} / \mathrm{V}$ ratio on $\varepsilon \in$ $[0,1]$ that is calculated by the exact formula (dashed line), by the third-order approximate formula (37) (solid line). Here we take: $e_{\delta}=3.8, \bar{e}_{\delta}=3.7, r_{\mu}=1, r_{v}=2.5$

Figs. 1, 2 present the third-order approximate curves and the exact curves of the Rayleigh wave $\mathrm{H} / \mathrm{V}$ ratio $\chi$ depending on $\varepsilon$ in the interval $[0,1]$. The approximate curves (solid lines) are calculated by Eq. (37) and the exact curves (dashed lines) are computed by using the exact secular equation (66) in Ref. [20], the exact expressions of displacements in Ref. [21] and the incompressible limit method [20]. It is shown from Figs. 1, 2 that the the third-order approximate formula (37) is a good approximation. Note that, an elegant approximate formula of $\mathrm{H} / \mathrm{V}$ ratio was obtained in [17] for the incompressible media. 
However, this formula is only good for high frequency, not for small frequency as shown in Fig. 5 of [2].

\section{Special cases:}

When $\varepsilon=0$, from (34) we have

$$
\chi=\left|\frac{\bar{A}_{0}}{\bar{B}_{0}}\right|=\left|\frac{h_{12}}{h_{11}}\right| .
$$

On the other hand, from (23) and $\Sigma(0)=0$ we have $|H|=0$, i.e.,

$$
h_{12}^{2}+h_{11} h_{22}=0
$$

From (40) and (41) it follows

$$
\chi^{2}=\frac{h_{12}^{2}}{h_{11}^{2}}=-\frac{h_{22}}{h_{11}}=b_{1} b_{2}=\sqrt{1-x} .
$$

When the layer and the half-space are both isotropic

$$
c_{11}=c_{22}, c_{66}=\mu, \bar{c}_{11}=\bar{c}_{22}, \bar{c}_{66}=\bar{\mu}, c_{11}-c_{12}=2 c_{66}, \bar{c}_{11}-\bar{c}_{12}=2 \bar{c}_{66} .
$$

With the help of (43) and (38) one can see that

$$
\begin{aligned}
& x=\frac{\rho c^{2}}{\mu}, e_{\delta}=\bar{e}_{\delta}=4, c_{2}=\sqrt{\frac{\mu}{\rho}}, \bar{c}_{2}=\sqrt{\frac{\bar{\mu}}{\bar{\rho}^{\prime}}}, r_{v}=\frac{c_{2}}{\bar{c}_{2}}, r_{\mu}=\frac{\bar{\mu}}{\mu}, \\
& \bar{x}=r_{v}^{2} x, S=2-x, P=1-x, b_{1}=1, b_{2}=\sqrt{1-x} .
\end{aligned}
$$

Then, the Rayleigh wave H/V ratio is defined by Eq. (37) with $\bar{A}_{i}$ and $\bar{B}_{i}(i=0,1,2,3)$ are given by

$$
\begin{aligned}
& \bar{A}_{0}=\frac{\sqrt{1-x}-1}{r_{\mu}}, \bar{A}_{1}=-\frac{x}{r_{\mu}}, \\
& \bar{A}_{2}=-4+\frac{4(1-\sqrt{1-x})}{r_{\mu}}-\frac{x+(x-4) \sqrt{1-x}}{r_{\mu}^{2}}, \\
& \bar{A}_{3}=-\frac{1+\sqrt{1-x}}{r_{\mu}}\left(r_{v}^{2} x+3 \sqrt{1-x}\right),
\end{aligned}
$$

and

$$
\begin{aligned}
& \bar{B}_{0}=\frac{1+\sqrt{1-x}}{r_{\mu}}, \bar{B}_{1}=4-r_{v}^{2} x-\frac{2(1-\sqrt{1-x})}{r_{\mu}}, \\
& \bar{B}_{2}=\frac{1+\sqrt{1-x}}{r_{\mu}}\left(2-r_{v}^{2} x+2 \sqrt{1-x}\right) \\
& \bar{B}_{3}=8-8 r_{v}^{2} x+r_{v}^{4} x^{2}+\frac{\left(3+r_{v}^{2}\right)(1-\sqrt{1-x})}{r_{\mu}}-\frac{2(x+(x-4) \sqrt{1-x})}{r_{\mu}^{2}} .
\end{aligned}
$$




\section{CONCLUSIONS}

In this paper, the propagation of Rayleigh waves in an incompressible orthotropic elastic half-space coated by a thin incompressible orthotropic elastic layer is investigated. An approximate formula for the Rayleigh wave $\mathrm{H} / \mathrm{V}$ ratio of third-order in terms of dimensionless thickness of the layer has been established by using the relations between the traction and displacement amplitude vectors of Rayleigh waves at two sides of the welded interface between the layer and the half-space. It is shown numerically that the obtained approximate formula is a good approximation. The obtained approximate formula can be employed as theoretical base for evaluating mechanical properties of thin films from measured values of the Rayleigh wave H/V ratio.

\section{ACKNOWLEDGMENTS}

The work was supported by the Vietnam National Foundation for Science and Technology Development (NAFOSTED) under grant number 107.02-2017.07.

\section{REFERENCES}

[1] M. Junge, J. Qu, and L. J. Jacobs. Relationship between Rayleigh wave polarization and state of stress. Ultrasonics, 44, (3), (2006), pp. 233-237. doi:10.1016/j.ultras.2006.03.004.

[2] P. G. Malischewsky and F. Scherbaum. Loves formula and H/V-ratio (ellipticity) of Rayleigh waves. Wave Motion, 40, (1), (2004), pp. 57-67. doi:10.1016/j.wavemoti.2003.12.015.

[3] P. G. Malischewsky, F. Wuttke, and A. Ziegert. The use of surface acoustic waves for nondestructive testing. Schriftenreihe Werkstoffwissenschaffen, 17, (2002), pp. 135-140. (in German).

[4] L. M. Munirova and T. B. Yanovskaya. Spectral ratio of the horizontal and vertical Rayleigh wave components and its application to some problems of seismology. Izvestiia Physics of the Solid Earth, 37, (9), (2001), pp. 709-716.

[5] F. Scherbaum, K. G. Hinzen, and M. Ohrnberger. Determination of shallow shear wave velocity profiles in the Cologne, Germany area using ambient vibrations. Geophysical Journal International, 152, (3), (2003), pp. 597-612. doi:10.1046/j.1365-246x.2003.01856.x.

[6] P. C. Vinh and R. W. Ogden. On formulas for the Rayleigh wave speed. Wave Motion, 39, (3), (2004), pp. 191-197. doi:10.1016/j.wavemoti.2003.08.004.

[7] P. C. Vinh and R. W. Ogden. Formulas for the Rayleigh wave speed in orthotropic elastic solids. Archives of Mechanics, 56, (3), (2004), pp. 247-265.

[8] R. W. Ogden and P. C. Vinh. On Rayleigh waves in incompressible orthotropic elastic solids. The Journal of the Acoustical Society of America, 115, (2), (2004), pp. 530-533. doi:10.1121/1.1636464.

[9] P. C. Vinh and R. W. Ogden. On the Rayleigh wave speed in orthotropic elastic solids. Meccanica, 40, (2), (2005), pp. 147-161. doi:10.1007/s11012-005-1603-6.

[10] P. C. Vinh and P. G. Malischewsky. An improved approximation of Bergmann's form for the Rayleigh wave velocity. Ultrasonics, 47, (1), (2007), pp. 49-54. doi:10.1016/j.ultras.2007.07.002.

[11] P. C. Vinh and P. G. Malischewsky. An approach for obtaining approximate formulas for the Rayleigh wave velocity. Wave Motion, 44, (7), (2007), pp. 549-562. doi:10.1016/j.wavemoti.2007.02.001.

[12] P. C. Vinh and P. G. Malischewsky. Improved approximations of the Rayleigh wave velocity. Journal of Thermoplastic Composite Materials, 21, (4), (2008), pp. 337-352. doi:10.1177/0892705708089479. 
[13] P. C. Vinh. On formulas for the velocity of Rayleigh waves in prestrained incompressible elastic solids. Journal of Applied Mechanics, 77, (2), (2010). doi:10.1115/1.3197139.

[14] P. C. Vinh and P. T. H. Giang. On formulas for the Rayleigh wave velocity in pre-strained elastic materials subject to an isotropic internal constraint. International Journal of Engineering Science, 48, (3), (2010), pp. 275-289. doi:10.1016/j.ijengsci.2009.09.010.

[15] P. C. Vinh. On formulas for the Rayleigh wave velocity in pre-stressed compressible solids. Wave Motion, 48, (7), (2011), pp. 614-625. doi:10.1016/j.wavemoti.2011.04.015.

[16] P. C. Vinh and P. G. Malischewsky. Improved approximations for the Rayleigh wave velocity in [-1, 0.5]. Vietnam Journal of Mechanics, 30, (4), (2008), pp. 347-358. doi:10.15625/0866$7136 / 30 / 4 / 5640$.

[17] A. E. H. Love. Some problems of geodynamics. Cambridge University Press, (1911).

[18] A. N. Stroh. Steady state problems in anisotropic elasticity. Studies in Applied Mathematics, 41, (1-4), (1962), pp. 77-103. doi:10.1002/sapm196241177.

[19] P. C. Vinh, N. T. K. Linh, and V. T. N. Anh. Rayleigh waves in an incompressible orthotropic half-space coated by a thin elastic layer. Archives of Mechanics, 66, (3), (2014), pp. 173-184.

[20] P. C. Vinh, V. T. N. Anh, and N. T. K. Linh. Exact secular equations of Rayleigh waves in an orthotropic elastic half-space overlaid by an orthotropic elastic layer. International Journal of Solids and Structures, 83, (2016), pp. 65-72. doi:10.1016/j.ijsolstr.2015.12.032.

[21] P. C. Vinh, V. T. N. Anh, and N. T. K. Linh. On a technique for deriving the explicit secular equation of Rayleigh waves in an orthotropic half-space coated by an orthotropic layer. Waves in Random and Complex Media, 26, (2), (2016), pp. 176-188. doi:10.1080/17455030.2015.1132859.

\section{APPENDIX A}

The expressions of $\bar{A}_{k}, \bar{B}_{k}(k=0,1,2,3)$

$$
\begin{aligned}
\bar{A}_{0}= & \frac{b_{1} b_{2}-1}{r_{\mu}}, \bar{A}_{1}=\frac{\left(b_{1}+b_{2}\right)\left(b_{1} b_{2}-1\right)}{r_{\mu}} \\
\bar{A}_{2}= & -\bar{e}_{\delta}+\frac{4\left(1-b_{1} b_{2}\right)}{r_{\mu}}-\frac{\left(1-b_{1} b_{2}\right)^{2}-b_{1} b_{2}\left(b_{1}+b_{2}\right)^{2}}{r_{\mu}^{2}} \\
\bar{A}_{3}= & \frac{\left(b_{1}+b_{2}\right)\left(4-\bar{e}_{\delta}-r_{v}^{2} x-3 b_{1} b_{2}\right)}{r_{\mu}} \\
\bar{B}_{0}= & \frac{b_{1}+b_{2}}{r_{\mu}}, \bar{B}_{1}=\bar{e}_{\delta}-r_{v}^{2} x-\frac{2\left(1-b_{1} b_{2}\right)}{r_{\mu}} \\
\bar{B}_{2}= & \frac{\left(b_{1}+b_{2}\right)\left(2 b_{1} b_{2}-2+\bar{e}_{\delta}-r_{v}^{2} x\right)}{r_{\mu}}, \\
\bar{B}_{3}= & \bar{e}_{\delta}^{2}-2 \bar{e}_{\delta}-2 \bar{e}_{\delta} r_{v}^{2} x+r_{v}^{4} x^{2}+\frac{\left(7-\bar{e}_{\delta}+r_{v}^{2} x\right)\left(1-b_{1} b_{2}\right)}{r_{\mu}} \\
& -2 \frac{\left(1-b_{1} b_{2}\right)^{2}-b_{1} b_{2}\left(b_{1}+b_{2}\right)^{2}}{r_{\mu}^{2}} .
\end{aligned}
$$

